\title{
Effect of Mass and Partial Slip on Boundary Layer Flow of a Nanofluid over a Porous Plate Embedded In a Porous Medium
}

\author{
Falana Ayodeji ${ }^{1}$; Alao Ahmed Abubakar ${ }^{2}$; Oladejo Oluyemisi Joy-Felicia ${ }^{3}$ \\ ${ }^{1,2}$ Department of Mechanical Engineering, University of Ibadan, Ibadan, Nigeria \\ ${ }^{3}$ Department of Civil Engineering, University of Ibadan, Ibadan, Nigeria
}

\begin{abstract}
This article is concerned with the effect of mass and partial slip on boundary layer flow of a nanofluid over a porous plate embedded in a porous medium. By taking suitable similarity variables, the governing partial differential equations are made dimensionless and then reduce to ordinary differential equations with the associated boundary conditions. The equations are then solved numerically using fourth order Runge-Kutta method.The results for the shear stress, the temperature distribution, the nanoparticle volume fraction, the skin friction coefficient, $f^{\prime \prime}(0)$, the reduced Nusselt number, $-\theta^{\prime}(0)$ and the reduced Sherwood number, $-\phi^{\prime}(0)$ are presented graphically illustrating the effects of mass slip, partial slip, permeability, thermophoresis, Brownian motion, Lewis number and Prandtl number. Our analysis reveals, among others, that skin friction increases with a decreasing mass slip and that the Nusselt number is a decreasing function, while the sherwood number is an increasing function of the thermophoretic number $N_{t}$. A comparison with previously published literatures on special case of the problem shows excellent agreement.
\end{abstract}

Keywords:Mass slip; Partial velocity slip; Permeable plate; Thermophoresis; Similarity variable

\section{Introduction}

The dynamics of fluids in porous media is generally dominated by the porous matrix which pervades the entire volume. A porous medium or a porous material is a material containing pores (voids). The skeletal portion of the material is often called the 'matrix' or 'frame'. Usually, pores are typically filled with a fluid (liquid or gas). Extremely small voids are called molecular interstices and very large ones are called caverns.Nanofluids have enormous potentials in enhancing heat transfer performances because of their thermophysical properties when compared to their conventional particle fluid suspensions. They have found applications inadvanced nuclear systems, biological sensors, hybrid power engines, fuel cells etc. Nanoparticles are made of oxides such as carbides, silica, alumina, copper oxides etc. Ahmad and Pop [1] studied mixed convection boundary layer flow from a vertical flat plate embedded in a porous medium filled with nanofluids. Nield and Kuznetsov [2] studied the problem proposed by Cheng and Minkowycz [3] about the natural convection past a vertical plate in a porous medium saturated by a nanofluid taking into account the Brownian motion and Thermophoresis. Bhattacharyya [4] investigated the effect of slip on boundary layer flow and mass transfer with chemical reaction over a permeable flat plate in a porous medium. Buongiorno [5] made a comprehensive survey of convective transport in nanofluids. He explained the enhanced heat transfer characteristics of nanofluids and their abnormal increase in thermal conductivities as a result of two main effects, namely, the Brownian diffusion and the thermophoretic diffusion of the nanoparticles. Buongiorno and $\mathrm{Hu}$ [6] investigated on the nanofluid coolants in advanced nuclear systems. Khan and Pop [7] used the model of Kuznetsov and Nield [8] to study the fundamental work on the boundary layer flow of nanofluid over a stretching sheet. Makinde and Aziz [9] extended the work of Khan and Pop [7] for convective boundary conditions.

Recently, Nur Husna Md. Yusoff et al. [10] studied the combined similarity-numerical solutions of MHD Boundary layer slip flow of non-Newtonian power-law nanofluids over a radiating moving plate. Moreover, Ali Pakravan and Yaghoubi [11] studied the combined effect of thermophoresis, Brownian motion and Dufour effects on natural convection of nanofluids. Lai and Kulacki [12] discussed the coupled heat and mass transfer by mixed convection from a vertical plate in a saturated porous medium. Hsu and Cheng [13] studied problems on Darcian and non-Darcian effects on a vertical plate natural convection in porous media. Kaviany [14] used the Darcy-Brinkman model to study the effects of boundary and inertia forces on forced convection over a fixed impermeable heated plate embedded in a porous medium. Kumari et al. [15] investigated the non-Darcian effects on forced convection heat transfer over a flat plate in a highly porous medium.

It is important to note that Darcy's classical flow model is a fundamental law relating the pressure gradient, viscosity and fluid velocity linearly through porous medium. Any deviation from Darcy's law and assumptions is termed as non-Darcy flow. Darcy's law works for creeping fluids of Reynolds number within a range of 1 to 10 Ishak et al. [16]. Most often, it is assumed that the upper limit of the applicability of Darcy's 
law is between $R e$ of 1 and 2 Bear [17]. The aim of this paper is to investigate mass and partial slip effect on boundary layer flow of a nanofluid over a porous plate embedded in a porous media.

However, to the best of author's knowledge, no attempt has been made to investigate the effect of mass and partial slip on boundary layer flow of a nanofluid over a porous plate embedded in a porous media. Hence, the problem is investigated. The governing partial differential equations are transformed to a system of ordinary differential equations using similarity approach and the resulting equations are then solved numerically using Runge-Kutta method. An investigation is carried out to illustrate the effect of various governing parameters viz., temperature, nanoparticle volume fraction, shear stress, skin friction coefficient, Nusselt and Sherwood number are discussed in details.

\section{Formulation of the Problem}

Consider a steady, two-dimensional, boundary layer flow of a nanofluid over a porous plate embedded in a porous medium. The $\mathrm{x}$-axis is taking along the surface of the porous plate and the $\mathrm{y}$-axis perpendicular to it. The temperature $T_{w}$ and concentration $C_{w}$ on the surface of the plate are kept constant, and assumed to be greater than the ambient temperature and concentration, $T_{\infty}$ and $C_{\infty}$, respectively. A physical geometry of the model is given in Fig 1 below. The governing equations of the flow field can be written in dimensional form as:

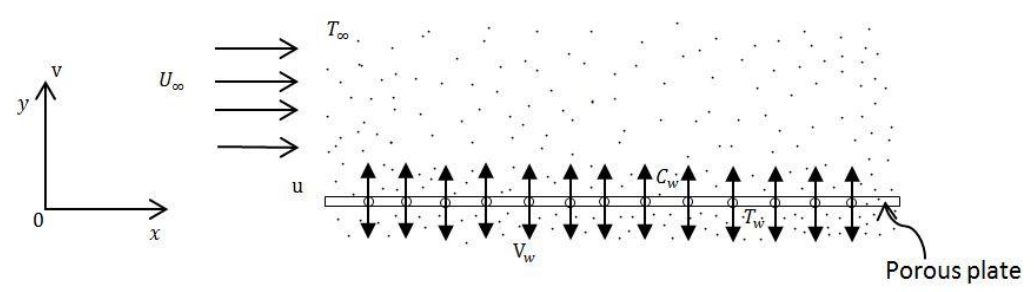

Fig: 1Geometry of the physical model

$\frac{\partial u}{\partial x}+\frac{\partial \mathrm{v}}{\partial y}=0$

$u \frac{\partial u}{\partial x}+\mathrm{v} \frac{\partial u}{\partial y}=v \frac{\partial^{2} u}{\partial y^{2}}-\frac{v}{k}\left(u-u_{\infty}\right)$

$u \frac{\partial T}{\partial x}+\mathrm{v} \frac{\partial T}{\partial y}=\alpha \frac{\partial^{2} T}{\partial y^{2}}+\tau\left[D_{B} \frac{\partial C}{\partial y}\left(\frac{\partial T}{\partial y}\right)+\frac{D_{T}}{T_{\infty}}\left(\frac{\partial T}{\partial y}\right)^{2}\right]$

$u \frac{\partial C}{\partial x}+\mathrm{v} \frac{\partial C}{\partial y}=D_{B}\left(\frac{\partial^{2} C}{\partial y^{2}}\right)+\frac{D_{T}}{T_{\infty}}\left(\frac{\partial^{2} T}{\partial y^{2}}\right)$

Where $\mathrm{u}$ and $\mathrm{v}$ are the velocity components in the $\mathrm{x}$ and $\mathrm{y}$ directions respectively, $u_{\infty}$ is the free stream velocity, $\mu$ is the viscosity, $T$ is the temperature of the nanofluid, $C$ is the concentration of the nanofluid, $T_{w}$ is the temperature along the porous plate, $C_{w}$ is the concentration along the porous plate, $\mathrm{T}_{\infty}$ and $\mathrm{C}_{\infty}$ are the ambient temperature and concentration respectively, $v=\frac{\mu}{\rho}$ is the kinematic viscosity, $\kappa=\kappa_{0} x$ is the Darcy permeability of the porous medium, $\mathrm{D}_{\mathrm{B}}$ is the Brownian motion coefficient, $\mathrm{D}_{\mathrm{T}}$ is the thermophoresis coefficient, $k$ is the thermal conductivity, $(\rho c)_{p}$ is the heat capacitance of the nanoparticles, $(\rho c)_{f}$ is the heat capacitance of the base fluid, $\alpha=\frac{k}{(\rho c)_{f}}$ is the thermal diffusivity, $\tau=\frac{(\rho c)_{p}}{(\rho c)_{f}}$ is the ratio between the heat capacitance of the nanoparticles and the heat capacitance of the base fluid.

The boundary conditions for velocity, temperature and concentration components are given by:

$u=U_{w}+\mathrm{L}_{1} \frac{\partial \mathrm{u}}{\partial \mathrm{y}}, \mathrm{v}=0, T=T_{w}, C=C_{w}+\mathrm{D}_{1} \frac{\partial \mathrm{C}}{\partial \mathrm{y}}$ at $y=0$

$u \rightarrow U_{\infty}, T \rightarrow T_{\infty}, C \rightarrow C_{\infty}$ as $y \rightarrow \infty$

Where $\mathrm{L}_{1}=L^{*}\left(R e_{x}\right)^{\frac{1}{2}}$, is the velocity slip factor and $\mathrm{D}_{1}=D^{*}\left(R e_{x}\right)^{\frac{1}{2}}$ is the mass slip factor with $L^{*}$ and $D^{*}$ being initial values of velocity and mass slip having dimension of length and $R e_{x}=\frac{U_{\infty} x}{v}$, is the local Reynolds number.

\section{Similarity Analysis}

The stream function $\psi(x, y)$ is introduce as $u=\frac{\partial \psi}{\partial y}$ and $v=-\frac{\partial \psi}{\partial x}$

For the expression in Eq. (6), the continuity equation Eq. (1) is satisfied. Using Eq. (6), the momentum equation Eq. (2), energy equation Eq. (3) and the concentration of specie equation Eq. (4) takes the following form 


$$
\begin{gathered}
\frac{\partial \psi}{\partial \mathrm{y}} \frac{\partial^{2} \psi}{\partial x \partial \mathrm{y}}-\frac{\partial \psi}{\partial x} \frac{\partial^{2} \psi}{\partial y^{2}}=v \frac{\partial^{3} \psi}{\partial y^{3}}-\frac{v}{\kappa}\left(\frac{\partial \psi}{\partial \mathrm{y}}-u_{\infty}\right) \\
\frac{\partial \psi}{\partial \mathrm{y}} \frac{\partial T}{\partial x}-\frac{\partial \psi}{\partial x} \frac{\partial T}{\partial y}=\alpha \frac{\partial^{2} T}{\partial y^{2}}+\tau\left[D_{B} \frac{\partial C}{\partial y}\left(\frac{\partial T}{\partial y}\right)+\frac{D_{T}}{T_{\infty}}\left(\frac{\partial T}{\partial y}\right)^{2}\right] \\
\frac{\partial \psi}{\partial \mathrm{y}} \frac{\partial C}{\partial x}-\frac{\partial \psi}{\partial x} \frac{\partial C}{\partial y}=D_{B}\left(\frac{\partial^{2} C}{\partial y^{2}}\right)+\frac{D_{T}}{T_{\infty}}\left(\frac{\partial^{2} T}{\partial y^{2}}\right)
\end{gathered}
$$

The boundary conditions in Eq. (5a) and (5b) for the velocity components reduce to

$$
\frac{\partial \psi}{\partial \mathrm{y}}=\mathrm{U}_{\mathrm{w}}+\mathrm{L}_{1} \frac{\partial^{2} \psi}{\partial \mathrm{y}^{2}}, \frac{\partial \psi}{\partial \mathrm{x}}=0 \text {, at } \mathrm{y}=0 ; \frac{\partial \psi}{\partial \mathrm{y}} \rightarrow \mathrm{U}_{\infty} \text { as } \mathrm{y} \rightarrow \infty
$$

We now introduce the dimensionless variables for $\psi, T$ and $C$ below

$$
\psi(\mathrm{x}, \mathrm{y})=\sqrt{\mathrm{u}_{\infty} v \mathrm{x}} f(\eta), \theta(\eta)=\left(\frac{\mathrm{T}-\mathrm{T}_{\infty}}{\mathrm{T}_{\mathrm{w}}-\mathrm{T}_{\infty}}\right) \text { and } \phi(\eta)=\left(\frac{\mathrm{C}-\mathrm{C}_{\infty}}{\mathrm{C}_{\mathrm{w}}-\mathrm{C}_{\infty}}\right)
$$

Where $\eta$ is the similarity variable and is defined as $\eta=y \sqrt{\frac{u_{\infty}}{v x}}$

From Eq. (11), we finally obtain the following self-similar equation as:

$f^{\prime \prime \prime}+\frac{1}{2} f f^{\prime \prime}-\kappa\left(f^{\prime}-1\right)=0$

$\frac{\theta^{\prime \prime}}{P r}+N b \phi^{\prime} \theta^{\prime}+N t\left(\theta^{\prime}\right)^{2}+\frac{1}{2} f \theta^{\prime}=0$

$\phi^{\prime \prime}+\frac{N t}{N b} \theta^{\prime \prime}+\frac{L e}{2} f \phi^{\prime}=0$

The boundary conditions Eq. (10) and (5) are transformed to the following forms:

$f=0, f^{\prime}=1+p f^{\prime \prime}, \theta=1, \phi=1+q \phi^{\prime}$ at $\eta=0$

$f^{\prime} \rightarrow 1, \theta \rightarrow 0, \phi \rightarrow 0$ as $\eta \rightarrow \infty$

Where $\mathrm{p}=\mathrm{L}^{*} \frac{u_{\infty}}{v}$, is the velocity slip parameterandq $=\mathrm{D}^{*} \frac{u_{\infty}}{v}$ is the mass slip parameter.

\section{Numerical Methods}

The coupled set of non-linear differential equations (12)-(4) along with the boundary conditions (15a) and (15b) form a three point BVP and does not form a closed form analytical solution. Therefore, it is solved numerically using fourth order classical 'Runge-Kutta Method'. In this method, we first convert the governing equations together with the boundary condition into first order system.

$$
\begin{gathered}
y_{1}^{\prime}=y_{2}, y_{2}^{\prime}=y_{3}, y_{3}^{\prime}=-\left[0.5 y_{1} y_{3}-\kappa\left(y_{2}-1\right)\right], \\
y_{4}^{\prime}=y_{5}, y_{5}^{\prime}=-P_{r}\left[N_{b} y_{5} y_{7}+N_{t} y_{5}^{2}+0.5 y_{1} y_{5}\right], \text { and } \\
y_{6}^{\prime}=y_{7}, y_{6}^{\prime}=-\left[0.5 L_{e} y_{1} y_{7}+N_{t b} y_{5}^{\prime}\right]
\end{gathered}
$$

Where $y_{1}=f, y_{2}=f^{\prime}, y_{3}=f^{\prime \prime}, y_{4}=\theta, y_{5}=\theta^{\prime}, y_{6}=\phi, y_{7}=\phi^{\prime}$

With the boundary conditions

$$
f(0)=0, f^{\prime}(0)=1+\mathrm{p} f^{\prime \prime}(0), \theta(0)=1 \text { and } \phi(0)=1+\mathrm{q} \phi^{\prime}(0)
$$

The method employed is the Adaptive Runge-Kutta method (Dormand-prince method) which is implemented in MATLAB as an m-file in the form of ode. It automatically finds the appropriate step size $\Delta \eta$ by comparing the results of a fourth-order and fifth-order method. It requires size function evaluations per $\Delta \eta$, and construct a fourth-fifth order method from these function evaluations.

\section{Results and Discussion}

\begin{tabular}{|c|c|c|c|c|}
\hline \multirow[t]{2}{*}{$N_{t}$} & \multicolumn{2}{|c|}{ Mania and Rama. [18] } & \multicolumn{2}{|c|}{ Present result } \\
\hline & $-\theta^{\prime}(0)$ & $-\phi^{\prime}(0)$ & $-\theta^{\prime}(0)$ & $-\phi^{\prime}(0)$ \\
\hline 0.1 & 0.952376 & 2.12939 & 0.9524 & 2.1294 \\
\hline 0.2 & 0.365357 & 2.51522 & 0.3654 & 2.5152 \\
\hline 0.3 & 0.135514 & 2.60881 & 0.1355 & 2.6088 \\
\hline 0.4 & 0.049465 & 2.60384 & 0.0495 & 2.6038 \\
\hline 0.5 & 0.017922 & 2.57310 & 0.0179 & 2.5731 \\
\hline
\end{tabular}

In other to find out the effect of mass and partial slip on the boundary layer flow of a nanofluid over a porous plate embedded in a porous medium, numerical computations are carried out. The results obtained are presented graphically to highlight salient features of the flow, the heat and the nanoparticle volume transfer characteristics. Table 1 shows the comparison of our results with Mania and Rama [18] and Table 2 shows our comparison with Khan and Pop [7], Anwar and Khan et al. [19] and Poornima and Bhaskar [20]. A good agreement is found and this supports the validity of our results for other cases.

Table 1: Comparisons of results for reduced Nusselt number $-\theta^{\prime}(0)$ and reduced Sherwood number $-\phi^{\prime}(0)$ with $L e=P_{r}=10, q=p=\kappa=0$ and $N_{b}=0.1$ 
From Figure 2 ( $a, b$ and $g$ ), the outcome of increase in permeability parameter, Lewis number and velocity slip on the dimensionless temperature is very small, and even with the continuous increase in these parameters, the decrease in temperature is almost unnoticed. Figure 2(c) shows the effect of mass slip on the dimensionless temperature in the flow field. The fluid temperature is maximum for $q=0$ (no mass slip). The increment of mass slip causes the temperature to decrease. But this decrease is very small and even upon continuous increase in mass slip, temperature decrease becomes unnoticed or died out at a certain level. Figure 2(d) interprets the effect of $N_{b}$ while Figure 2(e) demonstrates the influence of $N_{t}$. A significant increase in temperature accompanies a rise in $N_{b}$ and $N_{t}$ from 0.1 through 0.2 to 0.5 , figures 2(d) and 2(e). With increasing diffusion in the regime, the boundary
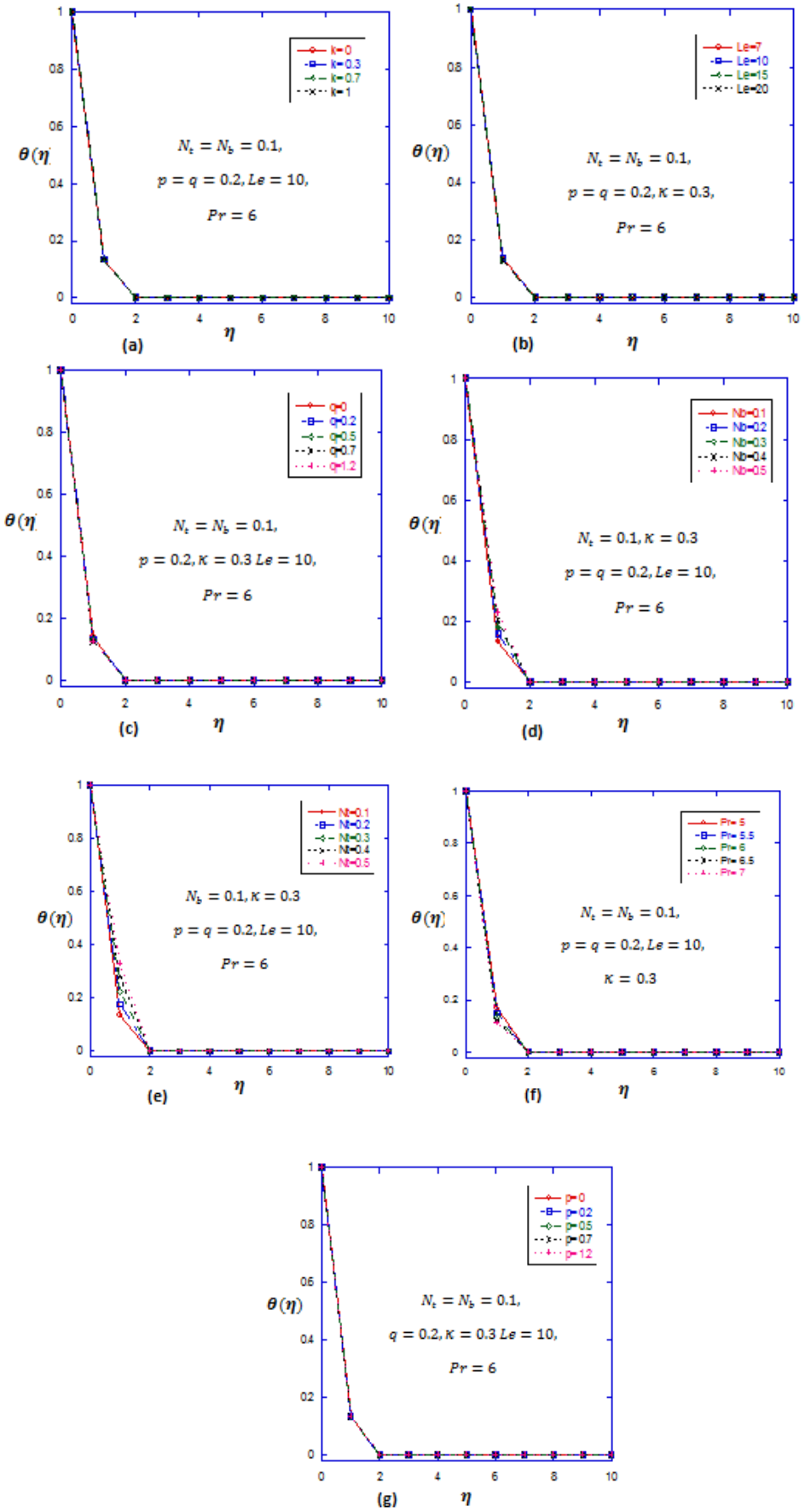

Fig. 2 Variations of dimensionless temperature profiles with $\kappa, L e, q, N_{b}, N_{t}, P_{r}$ and $p$

Layer gets heated which causes the thermal boundary layer thickness to increase. Figure 2(f) displays the influence of $P_{r}$ on the dimensionless temperature. Temperature is found to have decrease as $P_{r}$ increases. The decrease in temperature is more pronounced for fluids with higher Prandtl number $P_{r}$ and 
hence increasing the rate of heat transfer. Fluids with lower Prandtl number $P_{r}$ have larger thermal diffusivity. Figure 3 ( $a, c$ and g) depicts the dimensionless Nano particles volume fraction profiles for various values of permeability parameter $\kappa$, velocity slip $p$ and Prandtl number $P_{r}$ respectively. It is observed from these figures that an increase or decrease in any of the parameters $\left(\kappa, p\right.$ and $\left.P_{r}\right)$ has no effect on the nanoparticles volume fraction especially at the boundary (wall) i.e. $\eta=0$ and regions far away from the free stream. The dimensionless nanoparticles volume fraction decreases with an increasing Lewis number $L e$ as seen in Figure 3(b). This can further be explained from the definition of Lewis number, since Lewis number is inversely proportional to the Brownian motion coefficient having a kinematic viscosity, $v$ i. e. $L e=\frac{v}{D_{B}}$. As a result, fluids with higher Lewis number will have lower nanoparticles volume fraction.
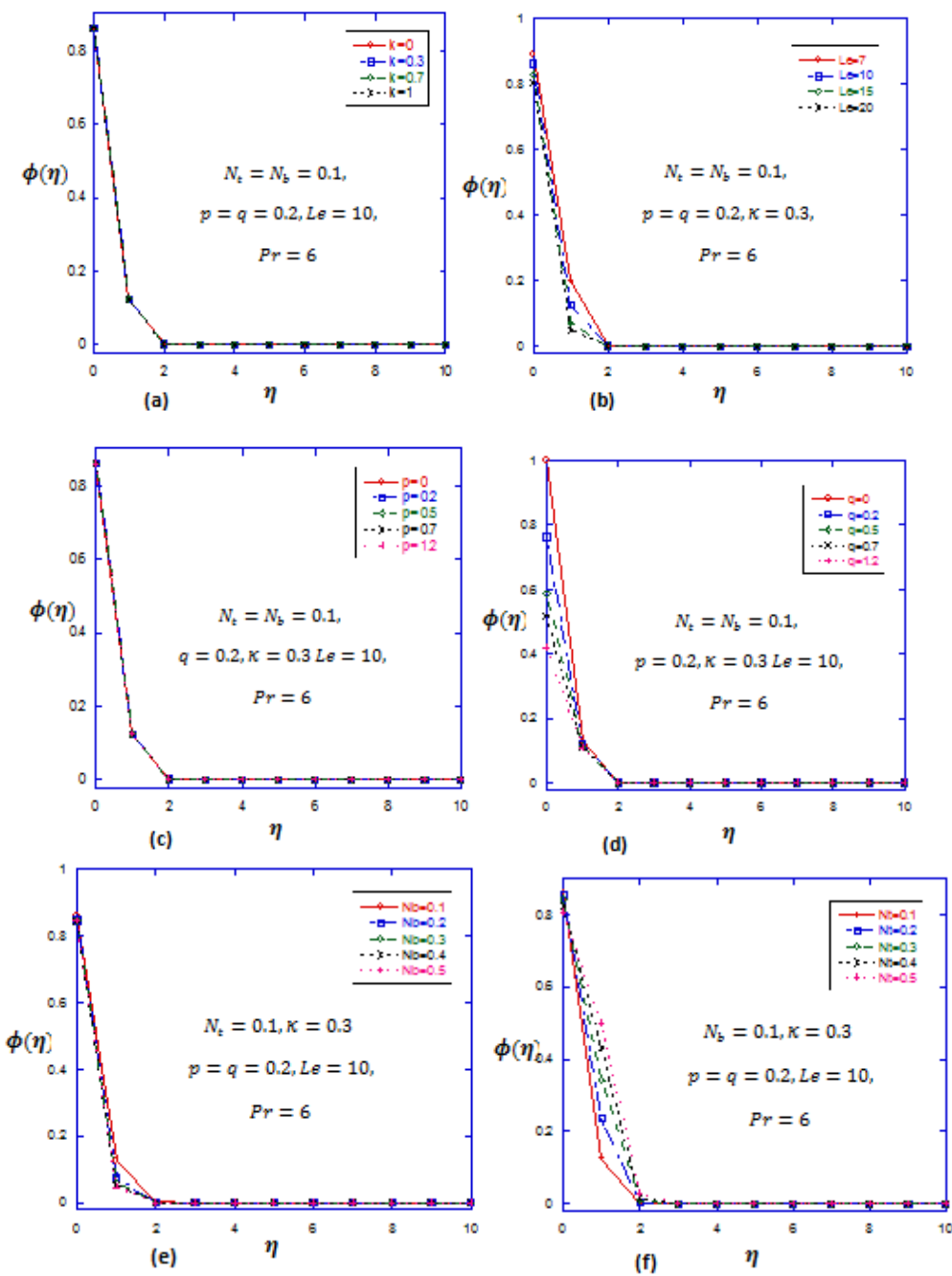

Figure 3(d) shows the effect of mass slip on the dimensionless nanoparticle volume fraction. As a result of mass slip on the boundary, the rate of mass transfer from the boundary of the plate to free stream reduces and the dimensionless solute decreases. From Figure 3(e) and 3(f), we note that the nanoparticle volume fraction profiles decreases with an increasing $N_{b}$ and it increases with an increase in $N_{t}$. Figure 4(a) depicts the dimensionless shear stress profiles for different values of permeability $\kappa$, the shear stress for a flat plate $(\kappa=0)$ is zero, but decreases at a boundary layer thickness of $\eta=1$ and $\eta=3$ (for plate with permeability of $\kappa=$ 0.3 and $\kappa=0.7$ respectively) then increases to a certain level and then decreases. Shear stress becomes zero at $\kappa=1$ (high permeability). The dimensionless shear stress decreases with an increasing Lewis number at a boundary layer thickness of $\eta=1$ (for fluids with $L e=7$ ) and $\eta=2$ (for fluids with $L e=10$ through 15 ) as seen in Figure 4(b). At higher Lewis number of $L e=20$, shear stress is zero. Figure 4(c). Illustrate the effect of Prandtl number $P_{r}$ on the dimensionless shear stress. Shear stress becomes zero for fluids with Prandtl number of $\left(P_{r}=6,6.5\right.$ through 7). Shear stress is $<0$ and $>0$ for fluids with Prandtl number of 5 and 5.5 respectively. As a result of the presence of velocity slip (p) on the wall of the plate, shear stress is zero over a boundary layer thickness of $\eta=2$ and then it increases and decreases towards free stream as seen in Figure 4(d). 


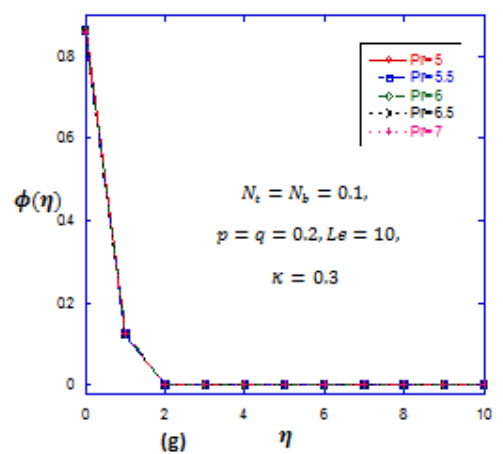

Fig. 3 Variations of dimensionless Nano particles volume fraction profiles with $\kappa, L e, q, N_{b}, N_{t}, P_{r}$ and $p$
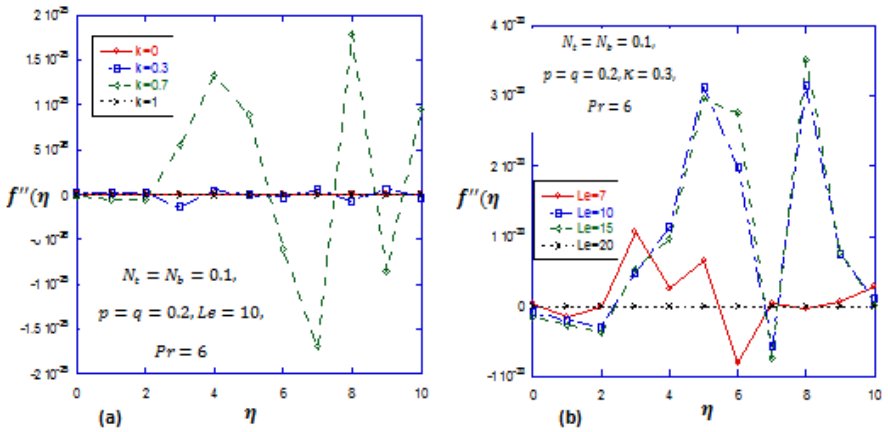

Figure 4(e). Demonstrate the influence of mass slip on the dimensionless shear stress. Shear stress is $<0$ for fluids with no mass slip and for fluids with mass slip of ( $\mathrm{q}=0.2$ through 0.5 to 1.2 ) over a boundary layer thickness of $\eta=2$, then it increases towards free stream. It is observed from Figure 4(f) that shear stress is zero for fluids with higher Brownian diffusion of $N_{b}=0.4$ through 0.5 , shear stress $<0$ over a boundary layer thickness of $\eta=2$ then it increases towards free stream for fluids with Brownian diffusion of $N_{b}=0.1,0.2$ and 0.3. In Figure 4(g), shear stress $<0$ for $N_{t}=1$ at $\eta=2$, then increases towards free stream. As $N_{t}$ increases from 0.2 , through 0.3 to 0.5 , shear stress becomes zero over a boundary layer thickness of $\eta=2$ then increases towards free stream. From Figure 5, skin friction at the boundary of the plate i.e. $\eta=0$ increases with decreasing mass slip, and as free stream is approached with an increasing slip velocity, skin friction decreases with an increasing mass slip.
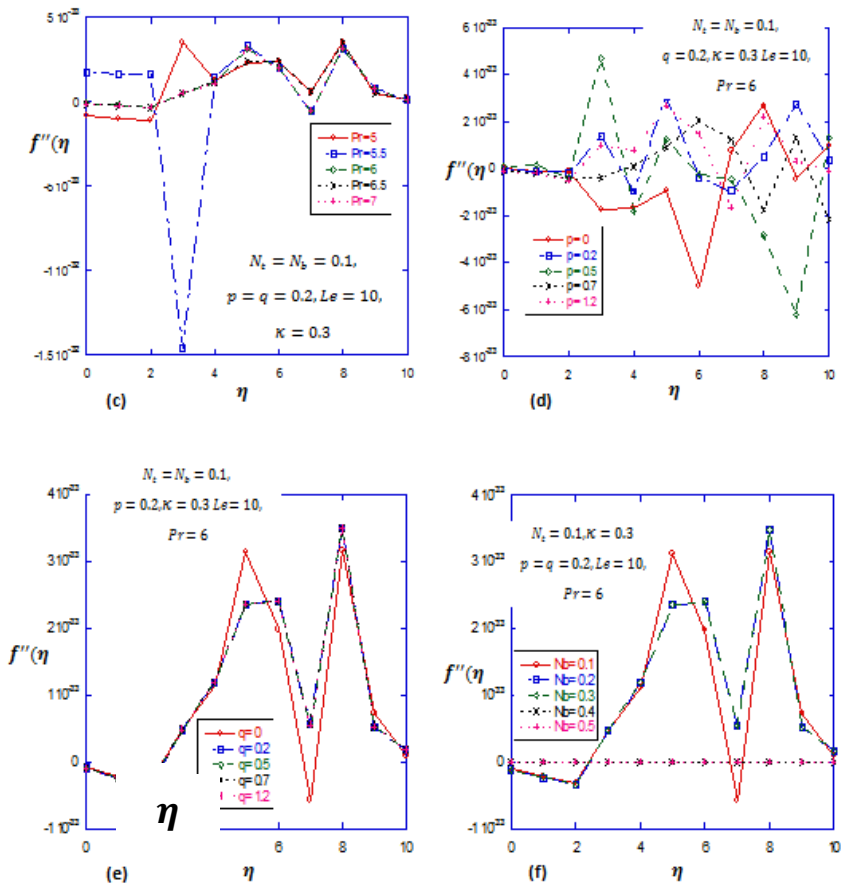

Fig. 4 Variations of dimensionless shear stress profiles with $\kappa, L e, P_{r}, p, q, N_{b}$, and $N_{t}$ 

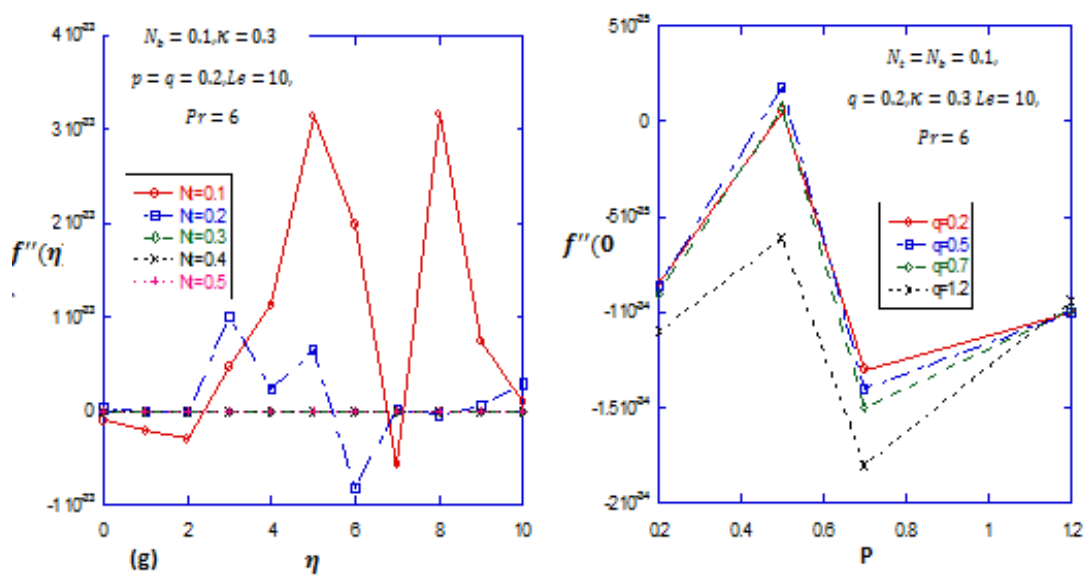

Fig. 5 Skin friction against $p$

Table 2: Comparisons of results for reduced Nusselt number $-\theta^{\prime}(0)$ and reduced Sherwood number $-\phi^{\prime}(0)$ with $L e=P_{r}=10, q=p=\kappa=0$

\begin{tabular}{|c|c|c|c|c|c|c|c|c|c|}
\hline$N_{b}$ & $N_{t}$ & \multicolumn{2}{|c|}{ Khan and Pop [7] } & \multicolumn{2}{c|}{ Anwar and Khan et al. [19] } & \multicolumn{2}{|c|}{ Poornima and Bhaskar. [20] } & Present result \\
\hline & & $-\theta^{\prime}(0)$ & $-\phi^{\prime}(0)$ & $-\theta^{\prime}(0)$ & $-\phi^{\prime}(0)$ & $-\theta^{\prime}(0)$ & $-\phi^{\prime}(0)$ & $-\theta^{\prime}(0)$ & $-\phi^{\prime}(0)$ \\
\hline 0.1 & 0.1 & 0.9524 & 2.1294 & 0.9524 & 2.1294 & 0.952376 & 2.12939 & 0.9524 & 2.1294 \\
\hline 0.2 & 0.2 & 0.3654 & 2.5152 & 0.3654 & 2.5152 & 0.365357 & 2.51522 & 0.3654 & 2.5152 \\
\hline 0.3 & 0.3 & 0.1355 & 2.6088 & 0.1355 & 2.6088 & 0.135514 & 2.60881 & 0.1355 & 2.6088 \\
\hline 0.4 & 0.4 & 0.0495 & 2.6038 & 0.0495 & 2.6038 & 0.049465 & 2.60384 & 0.0495 & 2.6038 \\
\hline 0.5 & 0.5 & 0.0179 & 2.5731 & 0.0179 & 2.5731 & 0.017922 & 2.57310 & 0.0179 & 2.5731 \\
\hline
\end{tabular}

\section{Concluding Remarks}

In summary, this study has numerically analyzed the effect of mass and partial slip on boundary layer flow of a nanofluid over a porous plate embedded in a porous medium. By using the similarity transformation approach, the governing partial differential equations are transformed into non-linear ordinary differential equations and the resulting problem is solved using Runge-Kutta method. The influence of the governing parameters on the heat and mass transfer has been closely examined. The following conclusions are drawn from the analysis:

1. Dimensionless temperature decreases slightly with increasing permeability $\kappa$, Lewis number $L e$, mass slip $q$ and velocity slip $p$. Dimensionless temperature also decreases with an increase in Prandtl number $P_{r}$ while a significant increase in $N_{b}$ and $N_{t}$ accompanies a rise in temperature.

2. At the boundary $(\eta=0)$, an increase or decrease in permeability $\kappa$, velocity slip $p$ and Prandtl number $P_{r}$ do not bring about any increase or decrease in the dimensionless nanoparticles volume fraction i.e. it is not affected by any of this parameters. The dimensionless nanoparticle volume fraction decreases with increasing Lewis number $L e$, mass slip $q$ and Brownian diffusion $N_{b}$ but increases with an increasing Thermophoresis $N_{t}$.

3. Skin friction with an increasing slip velocity $p$, increases with a decreasing mass slip $q$.

4. The permeability $\kappa$, Lewis number $L e$ and velocity slip $p$ have no effect on the heat transfer. An increase in mass slip $q$ and Prandtl number $P_{r}$ is accomplish with an increase in heat transfer while an increase in $N_{b}$ and $N_{t}$ causes a decrease.

5. The permeability $\kappa$, velocity slip $p$ and Prandtl number $P_{r}$ do not affect the rate of mass transfer. At the boundary $(\eta=0)$, the rate of mass transfer decreases significantly with increasing mass slip $q$ and Lewis number $L e$. Mass transfer also decreases with an increasing $N_{t}$ and increases with an increasing $N_{b}$.

\section{Nomenclature}

C Nanoparticle volume fraction

$C_{w} \quad$ Nanoparticle volume fraction on the plate

$C_{\infty} \quad$ Nanoparticle volume fraction in the free stream

$N u_{x} \quad$ Nusselt number

$R e_{x} \quad$ Local Reynolds number

$T$ Temperature of the fluid

$T_{w} \quad$ Temperature of the plate

$D \quad$ Diffusion coefficient

$D_{1} \quad$ Mass slip factor 
$D a_{x} \quad$ Local Darcy number

$L \quad$ Reference length

$L_{1} \quad$ Velocity slip factor

$\kappa \quad$ Permeability parameter

$P_{r} \quad$ Prandtl number

Le Lewis number

$N_{t} \quad$ Thermophoresis parameter

$N_{b} \quad$ Brownian parameter

$U_{\infty} \quad$ Free stream velocity

$U_{w} \quad$ Wall velocity

$u \quad$ Velocity component in $\mathrm{x}$-direction

$v \quad$ Velocity component in y-direction

$x \quad$ Distance along the plate

$y \quad$ Distance perpendicular to the plate

Greek Symbols

$p \quad$ Velocity slip parameter

$\eta \quad$ Similarity variable

$q \quad$ Mass slip parameter

$\mu \quad$ Coefficient of fluid viscosity

$v \quad$ Kinematic fluid viscosity

$\phi \quad$ Dimensionless nanoparticle volume fraction

$\rho \quad$ Fluid density

$\psi \quad$ Stream function

\section{References}

[1]. Ahmad S and Pop I. (2010). International communication in Heat and Mass Transfer, 37, 987-991.

[2]. Nield D A and Kuznetsov A V. (2009). International journal of Heat and Mass Transfer, 52(25-26), 5792-5795

[3]. Cheng P and Minkowycz W J. (1977). Journal of Geophysical research, 2040-2044.

[4]. Bhattacharyya Krishnendu . (2012). Slip effects on Boundary layer flow and mass transfer with chemical reaction over a permeable flat plate in a porous medium. Frontiers in Heat and Mass Transfer.

[5]. Buongiorno, J. (2006). Convective transport in nanofluids. ASME journal of Heat Transfer, 128, 240-250.

[6]. Buongiorno J, Hu. (2005). Nanofluid coolants for advanced nuclear power plants. Proceedings of ICAPP, 15-19.

[7]. Khan W A and Pop I. (2010). International journal of Heat and mass transfer, 53(11-12), 2477-2483.

[8]. Kuznetsov V A and Nield D A. (2010). International Journal of Thermal Sciences, 49(2), 243-247.

[9]. Makinde O D and Aziz A. (2011, July). Boundary layer flow of a nanofluid past a stretching sheet with a convective boundary condition. International Journal of Thermal Sciences, 50, 1326-1332.

[10]. Nur Husna Md. Yusoff, Md. Jashim Uddin* and Ahmad Izani Md. Ismail. (2014). Combined Similarity- numerical Solutions of MHD Boundary Layer Slip Flow of Non-Newtonian Power-law Nanofluids over a Radiating Moving Plate. Sains Malaysiana, 151159

[11]. Ali Pakravan Hossein and Mahmood Yaghoubi. (2010). International Journal of Thermal Sciences, 1-9.

[12]. Lai F C and Kulacki F A. (1991). Non-Darcy Mixed Convection along a vertical wall in a saturated porous medium. International Journal of Heat and Mass Transfer, 113, 252-255.

[13]. Hsu C T and Cheng P. (1985). The Brinkman model for natural convection about a semi-infinite vertical plate in a porous medium. International Journal of Heat and Mass Transfer, 28, 683-697.

[14]. Kaviany M,. (1987). Boundary-layer treatment of forced convection heat transfer from a semi-infinite flat plate embedded in porous media. American Society of Mechanical Engineering Journal of Heat Transfer, 109, 345-349.

[15]. Kumari M, Pop I and Nath G. (1990). Non-Darcian effects on forced convection heat transfer over a flat plate in a highly porous medium. Acta Mech, 84, 201-207.

[16]. Ishak A, Nazar R and Pop I. (2006). Steady and unsteady boundary layers due to a stretching vertical sheet in a porous medium using Darcy-Brinkman equation model. International Journal of Applied Mechanical Engineering, 11(3), 623-637.

[17]. Bear J. (1972). Dynamics of Fluids in porous media. Dover, New york.

[18]. Mania Goyal and Rama Bhargava. (2013). Numerical solution of MHD viscoelastic Nanofluid flow over a stretching sheet with partial slip and Heat source/sink. journal of heat transfer, 11 pages.

[19]. Anwar M. I., Khan I., Sharidan S. and Salleh M. Z. (2012). Conjugate effects of heat and mass transfer of nanofluids over a nonlinear stretching sheet. International journal of physical science, 4081-4092.

[20]. Poornima and Bhaskar. (2013). Radiation effects on MHD free convective boundary layer flow of nanofluids. Advances in Applied Science Research, 4, 190-202. 